Revue de droit comparé du travail et de la sécurité sociale

$3 \mid 2019$

Les migrations internationales de travail

\title{
Protéger les droits des travailleurs migrants : un défi pour le droit du travail ?
}

\section{Kübra Dogan Yenisey}

\section{(2) OpenEdition}

1 Journals

Édition électronique

URL : https://journals.openedition.org/rdctss/1454

DOI : $10.4000 /$ rdctss. 1454

ISSN : 2262-9815

Éditeur

Centre de droit comparé du travail et de la sécurité sociale

Édition imprimée

Date de publication : 1 novembre 2019

Pagination : 12-25

ISSN : 2117-4350

\section{Référence électronique}

Kübra Dogan Yenisey, «Protéger les droits des travailleurs migrants : un défı pour le droit du travail ?», Revue de droit comparé du travail et de la sécurité sociale [En ligne], 3 | 2019, mis en ligne le 01 novembre 2021, consulté le 12 novembre 2021. URL : http://journals.openedition.org/rdctss/1454 ; DOI : https:// doi.org/10.4000/rdctss. 1454

\section{(c) (†) $९$}

Revue de droit comparé du travail et de la sécurité sociale est mise à disposition selon les termes de la Licence Creative Commons Attribution - Pas d'Utilisation Commerciale - Pas de Modification 4.0 International. 


\title{
PROTÉGER LES DROITS DES TRAVAILLEURS MIGRANTS : UN DÉFI POUR LE DROIT DU TRAVAIL?
}

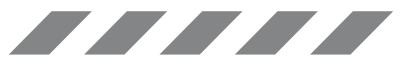

\begin{abstract}
It is hard to tell that labour laws, in general, have developed particular responses towards the problems of migrant workers. An overlook of international treaties reveal two parameters which directly affect the rights of migrants: the voluntary/forced nature of migration and the regularity. The primary aim of this report is to give a general picture of the legal status of migrant workers and show the challenges that labour law face while regulating their rights.
\end{abstract}

KEY WORDS: Migration, Migrant Workers, Labor Law.

\section{RÉSUMÉ}

Il est difficile de dire que le droit du travail, en général, a développé des réponses particulières à l'égard des problèmes des travailleurs migrants. Une abstraction des traités internationaux révèle deux paramètres qui affectent directement les droits des migrants en droit du travail: le caractère volontaire/forcé de la migration et la régularité. Le but premier de ce rapport est de donner une vue générale du statut juridique des travailleurs migrants et de montrer les défis auxquels le droit du travail doit faire face tout en définissant leurs droits.

MOTS CLÉS: Migration, travailleurs migrants, droit du travail. 


\section{Protéger LES DROITS DES TRAVAILLEURS MIGRANTS}

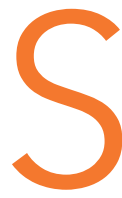

i la migration humaine n'est pas un phénomène nouveau, son ampleur et sa rapidité conduisent les chercheurs à l'étudier encore de nos jours. Les efforts entrepris aux niveaux international et régional visent principalement à élaborer une politique migratoire active, capable d'assurer un développement pérenne et une gestion efficace des mobilités transnationales'.

Au niveau régional, il revient à l'Union européenne d'avoir élaboré le premier modèle des migrations en provenance des pays tiers. L'Union africaine (UA) a, pour sa part, adopté un cadre de politique migratoire en 2006. Un document de programme de I'Union africaine sur la gouvernance des migrations de main-d'œuvre pour le développement, publié en 2017, énonce des principes pour renforcer la gouvernance et la réglementation des migrations de main-d'œuvre. En outre, la Communauté Economique de l'Union des États de l'Afrique de l'Ouest (CEDEAO) a créé une région intégrée avec une libre circulation des personnes qui constitue l'un des piliers de la Communauté économique africaine. La Commission de la CEDEAO a adopté une approche commune sur les migrations, le 18 janvier 2008, énonçant - entre autres- les principes de la libre circulation des personnes, de la lutte contre la traite des êtres humains, et de la protection des droits des migrants, des réfugiés et des demandeurs d'asile. Enfin, la Communauté de Développement de l'Afrique Australe (SADC) a adopté le cadre politique sur les migrations de main-d'œuvre en $2014^{2}$.

En Asie, I'Association des Nations de l'Asie du Sud-Est a adopté la Déclaration de Cebu sur la protection et la promotion des droits des travailleurs migrants en 2007. Dans la Déclaration de Katmandou en 2014, les États membres de l'Association Sud-Asiatique de Coopération Régionale ont accepté de s'engager « à collaborer et à coopérer pour une gestion sûre, ordonnée et responsable des migrations de main-d'œuvre en provenance

1 Ce travail est une synthèse du rapport préparé pour le Congrès mondial de l'ISLSSL (Torino 2018) et publié sous le titre «Safeguarding the Rights of Migrant Workers: Another Challenge for Labour Law?" ", in G. Casale et T. Treu (dir.), Transformations of Work - Challenges for Institutions and Social Actors, Bulletin of Comparative Labour Relations, Kluwer Law International, 2019, p. 227. Je souhaite remercier F. Columbu (Brésil), K. Koldinska (République Tchèque), C. Hayakaw (Japon), E. Binay (Afrique du Sud), F. Camas Roda (Espagne) et P. Herzfeld Olsson (Suède) qui ont préparé les rapports nationaux, ainsi que Z. Ulasan, étudiante à l'Université de Bilgi, pour ses contributions à la traduction du texte de l'anglais en français. En ce qui concerne la migration et le développement durable, voir ILO, Promoting fair migration, Report of the Committee of Experts on the Application of Conventions and Recommendations (art. 19, 22 and 35 of the Constitution), International Labour Conference, 105th Session, 2016, para. 29-43.

2 Voir E. Biney, "Labour migration as a social protection mechanism in Sub-Saharan Africa: Reflections on the EAC and SADC », Research paper for ISLSSL, 2018. 
d'Asie du Sud, afin d'assurer la sécurité et le bien-être de leurs travailleurs migrants dans les pays de destination en dehors de la région ». La communauté des Caraïbes a énoncé, dans la Déclaration de La Havane de 2016, son engagement profond pour le respect des droits de l'homme à l'égard des migrants, quel que soit leur statut migratoire.

Force est de constater que, malgré la multitude de documents politiques, il existe peu d'instruments juridiques contraignants régissant les droits des travailleurs migrants. En dépit de la nature largement débattue du phénomène, la migration est en général perçue comme hors du champ d'application du droit du travail. Toutefois, chaque immigrant est un candidat potentiel pour l'emploi sur un marché du travail national. Costello et Freedland montrent bien l'intersection et l'interaction entre les migrations et le droit du travail ${ }^{3}$.

De même, si le terme "travailleur migrant» peut englober différentes catégories de travailleurs, il revêt néanmoins un certain flou lorsqu'il s'agit de tracer ses frontières. L'article 11 de la Convention n 97 de l'OIT sur les travailleurs migrants de 1949 (révisée) définit le terme "travailleur migrant " comme "une personne qui émigre d'un pays vers un autre pays en vue d'occuper un emploi autrement que pour son propre compte; ce qui inclut toute personne admise régulièrement en qualité de travailleur migrant». La même définition est donnée par l'article 11 de la Convention n 143 sur les travailleurs migrants de 1975. La Commission d'experts déclare que la Convention n 97 s'applique aux migrants internationaux en vue d'occuper un emploi, aux réfugiés et aux personnes déplacées, ainsi qu'aux membres de la famille des travailleurs migrants ${ }^{4}$.

L'article 2 paragraphe 1 de la Convention internationale sur la protection des droits de tous les travailleurs migrants et des membres de leur famille prévoit que «l'expression «travailleurs migrants » désigne les personnes qui vont exercer, exercent ou ont exercé une activité rémunérée dans un État dont elles ne sont pas ressortissantes». Quelle que soit la motivation principale pour immigrer, la Convention considère l'engagement dans une activité rémunérée comme un élément de base pour définir le travailleur migrant.

Le but premier de cette contribution est de donner un aperçu général du statut juridique des travailleurs migrants (I), de montrer les défis auxquels le droit du travail (II) doit faire face et apporter des réponses (III). Ce rapport ne tente pas une analyse exhaustive de tous les problèmes, il se concentre sur plusieurs des questions majeures qui illustrent la complexité de la réglementation des travailleurs migrants.

\section{I - ANALYSE DU STATUT JURIDIQUE DES TRAVAILLEURS MIGRANTS}

S'agissant des instruments internationaux relatifs au droit des migrants, il convient de rappeler la Déclaration sur les droits de l'homme relative aux personnes non-ressortissantes du pays dans lequel elles vivent, la Convention des Nations Unies sur la protection des droits de tous les travailleurs migrants et des membres de leur famille, la Charte sociale européenne de 1961 et la Charte sociale révisée, sans nier l'importance des instruments internationaux généraux de la protection des droits des migrants ${ }^{5}$. Par ailleurs, I'OIT a

3 C. Costello, M. Freedland, "Migrants at Work, Immigration and Vulnerability in Labour Law», Oxford University Press, 2014.

4 Comm. Exp., Conv. n 97, Kyrgyzstan, 2016, 106th ILC Session 2017.

5 Voir B. Ryan, V. Mantouvalou, "The Labour and Social Rights of Migrants in International Law», in R. Rubio-Marin (dir.), Human Rights and Migration, 2014, p. 177. 
adopté de nombreux instruments, telles la Convention $n^{\circ} 97$, la Convention $n^{\circ} 143$ et les Recommandations $\mathrm{n}^{\circ} 86$ et $\mathrm{n}^{\circ} 151$.

Un aperçu général des traités internationaux révèle deux paramètres qui affectent directement les droits des migrants: le caractère volontaire/forcé de la migration et la régularité. Quatre catégories différentes doivent être couvertes du point de vue du droit du travail:

1 - Migrants forcés en situation régulière;

2- Migrants forcés en situation irrégulière;

3- Migrants en vue d'occuper un emploi et en situation régulière;

4- Migrants en vue d'occuper un emploi et en situation irrégulière.

\section{A - LES CATÉGORIES DE MIGRANTS}

\section{1 - Les migrants forcés}

Les migrants déplacés pour des raisons humanitaires constituent une part importante de la population migrante dans certains pays. L'Organisation Internationale pour les Migrations définit la migration forcée comme « un mouvement migratoire dans lequel existe un élément de coercition, y compris les menaces à la vie et aux moyens de subsistance, qu'elles résultent de causes naturelles ou soient d'origine naturelle $»^{6}$.

Dans les systèmes juridiques, il existe trois catégories différentes de migrants forcés: les réfugiés et les demandeurs d'asile, les bénéficiaires de la protection complémentaire/ subsidiaire et les bénéficiaires de la protection temporaire.

Le statut de réfugié impose aux États-nations le devoir d'admettre des étrangers sur leur territoire. L'article premier de la Convention de Genève définit le réfugié comme « craignant avec raison d'être persécuté du fait de sa race, de sa religion, de sa nationalité, de son appartenance à un certain groupe social ou de ses opinions politiques, [qui] se trouve hors du pays dont il a la nationalité et qui ne peut ou, du fait de cette crainte, ne veut se réclamer de la protection de ce pays, ou qui, s'il n'a pas de nationalité et se trouve hors du pays dans lequel il avait sa résidence habituelle à la suite de tels événements, ne peut ou, en raison de ladite crainte, ne veut y retourner ». Ainsi, la Convention restreint les motifs de persécution sur lesquels se fonder.

La Convention régissant les aspects spécifiques des problèmes des réfugiés en Afrique donne une définition plus large du statut de réfugié que celle de la Convention de Genève ${ }^{7}$. La Déclaration de Carthagène recommande ainsi l'utilisation dans la Région d'une définition ou d'un concept de refugié qui «s'étende aux personnes qui ont fui leur pays parce que leur vie, leur sécurité ou leur liberté étaient menacées par une violence généralisée, une agression étrangère, des conflits internes, une violation massive des droits de l'homme ou d'autres circonstances ayant perturbé gravement l'ordre public ». Par conséquent, la définition du concept de réfugié, comme la procédure de demande d'asile, présentent de grandes divergences dans le monde. En vertu de la Convention de Genève,

\section{6 https://www.iom.int/key-migration-terms}

7 Voir A. Abbas, D. Mystris, "The African Union Legal Framework for Protecting Asylum Seekers », in A. Abass et F. Ippolito (dir.), Regional Approach to the Protection of Asylum Seekers An International Legal Perspective, $2^{\mathrm{e}}$ ed., 2016, p. 19. 
les réfugiés (ou demandeurs d'asile) qui « résident régulièrement » bénéficient du droit d'exercer un emploi rémunéré (article 17).

En ce qui concerne l'accès au marché du travail des bénéficiaires de la protection complémentaire/subsidiaire et temporaire, il existe une protection internationale plus faible. La Directive 2004/83/CE de 2004 et la Directive 2011/95/UE de 2011 dite « qualification» énoncent les principes relatifs à ceux qui sont exclus du statut de réfugié. La personne éligible à la protection subsidiaire désigne le ressortissant d'un pays tiers qui n'est pas considéré comme un réfugié, mais pour lequel des motifs substantiels ont été démontrés et laissent à croire que la personne concernée, en retournant dans son pays d'origine, serait confrontée au risque réel de subir un préjudice grave.

La Convention de Genève, comme la Directive dite qualification, exigent une évaluation du statut individuel, ce qui ne répond pas à la nécessité urgente de protéger les nombreuses personnes qui fuient les violations des droits de l'homme et les conflits armés dans leurs pays d'origine. Ainsi, la Directive sur la protection temporaire (2002/55/ CE) adoptée le 20 juillet 2001 a élargi la définition étroite de réfugié. Ainsi, les 3,1 millions de personnes déplacées de Syrie qui ont traversé la frontière dans un afflux massif sont sous le statut de protection temporaire en Turquie.

En dehors des demandeurs d'asile, les personnes qui bénéficient d'un statut de protection internationale ne bénéficient d'aucun droit au travail pendant le processus de reconnaissance et les bénéficiaires de la protection complémentaire et temporaire disposent d'un accès très limité au marché du travail.

\section{2 - Les migrations aux fins de l'emploi}

Les personnes migrant en vue d'occuper un emploi dans un autre État peuvent être divisées en deux catégories: celles qui ont un permis de séjour permanent, et celles qui ont un permis de travail temporaire. La vulnérabilité des deux groupes et les exigences de protection varient d'un groupe à l'autre.

Un regard général sur les migrations en vue d'occuper un emploi montre une diversité des instruments juridiques internationaux et une multitude de modèles strictement régulés au niveau national. La communauté internationale élabore de nouveaux instruments tout en essayant de faire face à la complexité des migrations transnationales ${ }^{8}$.

S'agissant des droits nationaux, la législation sur les migrations détermine différentes catégories de migrants dont les voies de migration sont clairement définies. En outre, de nombreux pays ont des programmes d'immigration du travail qui se réfèrent à un ensemble de politiques réglementant l'admission, l'emploi et les droits des travailleurs migrants.

Un aperçu global des différents modèles législatifs révèle que les États-nations combinent des mesures pour contrôler l'immigration avec celles du statut juridique des immigrants dans les pays d'accueil. Les droits des migrants varient d'une région à l'autre. Le paramètre déterminant affectant la portée des droits des migrants semble être le niveau de compétence professionnelle des immigrés. Les conditions d'admission et les régimes de permis de travail sont en effet plus favorables pour les migrants hautement qualifiés que

8 P. Wickramasekara, «Bilateral agreements and memoranda of understanding on the migration of low-skilled workers: a review», ILO, ILC-Geneva, 2015, p. 13. 
pour les migrants peu qualifiés9. La comparaison de la Directive européenne sur les cartes bleues et de la Directive de l'UE sur les travailleurs saisonniers conduit à des conclusions similaires ${ }^{10}$.

\section{B - LES MIGRATIONS ET LA « RÉGULARITÉ »: ENTRE DROIT DU TRAVAIL, DROIT MIGRATOIRE ET DROIT DES DROITS DE L'HOMME}

\section{1 - Irrégularité juridique et validité du contrat de travail}

Les migrants illégaux sans papiers ou irréguliers - trois termes véhiculant des connotations différentes - ont des significations qui se chevauchent. Même si les notions de migration illégale/irrégulière/sans papiers occupent une place importante dans les programmes politiques, il n'existe pas de définition juridique claire. Les deux Conventions de I'OIT $n^{\circ} 143$ et $n^{\circ} 97$ se réfèrent aux critères d'«admission régulière ». Toutefois, la Convention des Nations Unies sur les travailleurs migrants retient une vision plus large de l'irrégularité: les travailleurs migrants sont considérés comme pourvus de papiers, ou en situation régulière, s'ils sont autorisés à entrer, séjourner et exercer une activité rémunérée dans l'État d'emploi (article 5/1). Prévoyant des normes minimales concernant les sanctions, la Directive 2009/52/CE définit l'« emploi illégal » comme «l'emploi d'un ressortissant de pays tiers en séjour irrégulier», et le "ressortissant d'un pays tiers en séjour irrégulier » comme le "ressortissant de pays tiers présent sur le territoire d'un État membre, qui ne remplit pas, ou n'accomplit plus, les conditions de séjour ou de résidence dans cet État membre $»^{11}$. Par conséquent, l'emploi illégal est lié aux conditions de séjour et de résidence.

L'irrégularité peut survenir à chaque étape du processus d'immigration : un non-national arrivant clandestinement sur le territoire de l'Etat d'accueil ; un étranger résidant légalement mais travaillant sans permis de travail; un étranger qui travaille malgré l'expiration de son visa. Toutes ces situations semblent entachées d'irrégularité. Il serait donc erroné de voir la migration irrégulière comme un phénomène social indépendant. L'illégalité de la migration a des liens étroits avec les politiques étatiques et revêt une dimension sociale, politique et juridique ${ }^{12}$. En outre, l'irrégularité présente une caractéristique d'ordre temporel dans la mesure où, après la date d'expiration du visa ou du permis de travail, la légalité de la relation contractuelle est remise en question. Costello démontre ainsi la « légalité à multiniveaux » et les variations temporelles du statut de migrants ${ }^{13}$.

La question essentielle consiste à déterminer si une irrégularité du processus migratoire - quelle qu'elle soit - a pour effet de rendre le contrat de travail invalide. II

9 M. Ruhs, The Price of Rights: Regulating International Labor Migration, 2015, p. 88.

10 P. Herzfeld Olsson, «Introduction to Directives», Bulletin of Comparative Labour Relations, National Effects of the Implementation of EU Directives on Labour Migration from Third Countries, 2016, p. 24.

11 Voir S. Peers, «Legislative Update: EC Immigration and Asylum Law Attracting and Deterring Labour Migration: The Blue Card and Employer Sanctions Directives», European Journal of Migration and Law, 11 (4), 2009, p. 387.

12 F. Duvell, "Paths into Irregularity: The Legal and Political Construction of Irregular Migration», European Journal of Migration and Law, 13, 2011, p. 275; B. Anderson, « Migration, immigration controls and the fashioning of precarious workers», Work, Employment and Society, vol. 24 (2), p. 312.

13 C. Costello, The Human Rights of Migrants and Refugees in European Law, 2015, p. 64. 
existe principalement deux types d'approches: les systèmes juridiques dans lesquels le contrat de travail conclu par un migrant en situation irrégulière est invalide et les systèmes juridiques dans lesquels l'irrégularité du statut migratoire fournit à l'employeur une cause pour mettre fin à la relation contractuelle. Compte tenu de la nécessité de protéger les migrants situation irrégulière, même si la norme juridique est considérée comme liée à l'ordre public, l'invalidité peut avoir un effet ex nunc. C'est le cas en Suède, en Turquie, au Brésil, en Espagne, alors qu'en République Tchèque c'est l'effet ex tunc.

S'agissant des indemnités liées à la résiliation de la relation contractuelle, si le contrat prend fin pour invalidité, les travailleurs migrants ne jouissent d'aucune indemnité découlant de la résiliation (en l'absence de règlement spécifique). Toutefois, dans certains pays comme la France, le législateur prévoit qu'en cas de résiliation du contrat, le migrant irrégulier sera éligible à une somme forfaitaire égale à un mois de salaire ${ }^{14}$.

Ces résultats démontrent que, dès lors qu'il existe une vulnérabilité et une exploitation dans une relation d'emploi, chaque système tente de prévenir ces injustices en se référant à différents outils juridiques. Néanmoins, sur le plan de l'efficacité, le modèle français semble le plus prévisible et offre plus de protection.

\section{La protection des migrants en situation irrégulière}

L'irrégularité dans le processus de migration a pour conséquence de priver les migrants de leurs droits découlant du contrat de travail. La jurisprudence de la Cour européenne des droits de l'homme a marqué une étape importante pour la protection des droits des migrants en situation irrégulière. Les arrêts Mendizabal ${ }^{15}$, Siliadin ${ }^{16}$ et Chowdury ${ }^{17}$ sont les premiers à s'y consacrer.

La Cour interaméricaine des droits de l'homme admet l'importance de l'accès à l'emploi pour les demandeurs d'asile. Ceux-ci, à l'instar des autres travailleurs migrants, acquièrent des droits du travail, indépendamment de leur statut - régulier ou irrégulier dans l'État d'accueil ${ }^{18}$. Dans un avis consultatif sur les droits des migrants sans papiers, la Cour interaméricaine fournit quelques lignes directrices, considérant que toute politique ou législation migratoire doit se conformer aux normes internationales et régionales en vigueur en ce qui concerne les restrictions aux droits de l'homme ${ }^{19}$.

14 Article L.8252-2 du Code du travail.

15 Aristimuno Mendizabal c. France, App. n 51431/99 (ECHR, 17 janvier 2006).

16 Siliadin v. France, App. $n^{\circ}$ 73316/01 (ECHR, 26 juillet 2005).

17 Chowdury and others v. Greece, App n² 21884/15 (ECHR, 30 mars 2017).

18 D. J. Cantor, S. Barichello, "Protection of Asylum Seekers under the Inter-American Human Rights System », in A. Abass et F. Ippolito (dir.), Regional Approach to the Protection of Asylum Seekers An International Legal Perspective, $2^{\mathrm{e}}$ ed., 2016, p. 287.

19 M. Ontiveros, "Migrant Labour in USA », in C. Costello et M. Freedland (dir.), Migrants at Work, op. cit., p. 189. 
À son tour, le Comité européen des droits sociaux ${ }^{20}$ a tenté d'élargir la protection offerte aux migrants en situation irrégulière. En réponse aux plaintes collectives déposées par la Fédération internationale des ligues des droits de l'homme ${ }^{21}$ et Defence of Children Internationa/22, le Comité a ainsi décidé d'élargir le champ d'application de la Charte sociale révisée à l'accès aux soins de santé d'urgence, au logement et à l'assistance sociale, préalables essentiels au maintien de la dignité humaine ${ }^{23}$.

\section{C - LES TRAVAILlEURS MIGRANTS SOUS DEUX PARAMÈTRES}

La réglementation du statut juridique des travailleurs migrants révèle une grande diversité, tant au niveau national qu'international, et offre une faible protection. Peu d'instruments internationaux sont consacrés au traitement des travailleurs migrants et leur faible taux de ratification témoigne de l'absence d'engagement international en faveur de leur protection. En outre, la reconnaissance formelle du statut de migrant en droit international ne confère pas, dans la plupart des cas, un droit au travail automatique dans les États d'accueil.

Au niveau national, les travailleurs migrants sont à l'intersection du droit des migrations, du droit du travail, du droit relatif aux droits de l'homme et du droit pénal. Chacune de ces disciplines, avec ses principes et ses objectifs (protection du travailleur, sanction, compensation), ses concepts et techniques (comme la charge de la preuve), réglemente une part du phénomène migratoire et $n$ 'interagit pas toujours harmonieusement.

Les régimes de visas et les programmes de migration dévoilent la tendance des politiques à se concentrer sur les compétences des migrants. La première étape de ce processus consiste à déterminer les emplois disponibles sur le marché du travail. La deuxième étape se focalise sur l'admission du migrant et le fait qu'il conserve le travail ciblé pendant son séjour. Par des accords bilatéraux, les États-nations contrôlent les postulants et les emplois recherchés. Étant donné que l'objectif est d'apporter une solution à la pénurie de main-d'œuvre sur le marché du travail, bon nombre de ces programmes sont temporaires et axés dans l'intérêt de l'entreprise ${ }^{24}$.

20 Conseil de l'Europe, Digest of the case law of the European Committee of Social Rights, 1 er septembre 2008, p. 183.

21 Defence of Children International v. Netherlands CC 47/2008, European Committee of Social Rights, 20 Octobrer 2009.

22 Ibid.

23 C. O'Cinneide, «Migrants Rights under the Social Charter », in C. Costello et M. Freedland (dir.), Migrants at Work, op. cit, p. 289.

24 J. Howe, R. J. Owens, "The Regulatory Challenges » (Introduction), Temporary Labour Migration in the Global Era, Bloomsbury Collections, 2016, p. 9. 
Le statut de l'immigrant détermine donc de façon significative la position du travailleur sur le marché du travail25. Le statut personnel d'un travailleur migrant conduit à la " division », telle qu'elle est appelée par Freedland et Costello, des sujets du droit du travail. La législation sur les migrations impose certains types de conditions de travail en créant différentes catégories de migrants ${ }^{26}$, et une ancienne discussion sur « du statut au contrat » semble resurgir avec les travailleurs migrants ${ }^{27}$.

En outre, la législation sur les migrations réglemente de manière détaillée l'accès au marché du travail et impose de nouvelles obligations aux employeurs pour empêcher le recrutement de migrants irréguliers. Les règles impératives et les infractions au droit des migrations font dès lors partie intégrante du droit du travail ${ }^{28}$. Le nombre croissant de ces règles laisse à penser que les lois sur les migrations instrumentalisent le droit du travail dans la lutte contre les migrations illégales. De plus, l'instrumentalisation du droit du travail affecte la mise en œuvre du principe de l'égalité entre les ressortissants et les non-ressortissants, principe fondateur qui règlemente le statut juridique des travailleurs migrants ${ }^{29}$.

De toute évidence, certains travailleurs migrants rencontrent davantage de difficultés que d'autres. Les groupes les plus vulnérables ne bénéficient d'aucune protection; parmi eux, les travailleurs domestiques ou les migrants qui travaillent dans l'agriculture sont les premiers concernés. Une approche sectorielle pourrait contribuer à réduire les vulnérabilités rencontrées par les migrants.

En ce qui concerne les droits collectifs des migrants en situation irrégulière, le Comité de la liberté syndicale a estimé que le déni de leurs droits était en contradiction avec l'article 2 de la Convention n 87 de l'OIT ${ }^{30}$. De même, l'article 11 de la CEDH peut également être interprété de manière à protéger le droit d'organisation des migrants irréguliers ${ }^{31}$. Néanmoins, des obstacles persistent et les empêchent de jouir pleinement de leurs droits.

25 N. Kountouris, «The Legal Determinants of Precariousness in Personal Work Relations: A European Perspective ", Comparative Labor Law and Policy Journal, 34, 2012, p. 27.

26 B. Anderson, "Migration, immigration controls and the fashioning of precarious workers ", Work, Employment and Society, vol. 24 (2), p. 300.

27 M. Freedland, C. Costello « Migrants at Work and the Division of Labour Law», Migrants at Work, op. cit.; C. Costello, "EU Migration and Asylum Law: A Labour Law Perspective », in A. Bogg, C. Costello, A.C.L. Davies (dir.), Research Handbook on EU Labour Law, 2016, p. 299.

28 L. Lamarche, "Human Rights, Social Security and Migrant Workers», Bulletin of Comparative Labour Relations, Social Security and Migrant Workers, 84, 2014, p. 9; A. Bogg, «Illegality, Public Policy, and the Contract of Employment », in A. Bogg, C. Costello, A.C.L. Davies, J. Prassl (dir.), The Autonomy of Labour Law, Bloomsbury Collections, 2015, p. 399.

29 B. Ryan, "Employer Checks of Immigration Status and Employment Law», in C. Costello et M. Freedland (dir.), Migrants at Work, op. cit., p. 243.

30 Affaire $n^{\circ} 2121$ (Spain), Digest of decisions and principles of the Freedom of Association Committee of the Governing Body of the ILO, ILO, 5e edition, 2006, para. 214; Affaire $n^{\circ} 2620$ (Republic of Korea), Report n 374.

31 V. Mantouvalou, "Organizing against Abuse and Exclusion The Associational Rights of Undocumented Workers », in C. Costello and M. Freedland (dir.), Migrants at Work, op. cit., p. 390. 


\section{II - « MUTUALISATION DES RISQUES » ET RÈGLEMENTATION DES MIGRATIONS}

Le phénomène migratoire consiste en des interactions complexes et dynamiques entre les politiques économiques, sociales et les réglementations juridiques. Fudge ${ }^{32}$ et Shamir ${ }^{33}$ démontrent combien il est important d'élaborer une approche globale du droit du travail pour lutter contre la traite des êtres humains et les migrations forcées. Si les droits de l'homme peuvent être efficaces pour réglementer les formes sévères d'exploitation du travail - telles que le travail forcé, la servitude ou l'esclavage - c'est en revanche le droit du travail qui dispose du plus large éventail d'outils ${ }^{34}$ pour corriger les inégalités sur le marché du travail.

La complexité du problème des migrations révèle des relations tendues entre le droit des migrations et le droit du travail ${ }^{35}$. Une approche fondée sur «la mutualisation et la démutualisation des risques " développée par Freedland et Kountouris ${ }^{36}$ peut nous permettre de mieux appréhender le phénomène, en recherchant une mutualisation équitable des risques qui servirait de base à une règlementation. Ainsi, la construction juridique des relations de travail repose sur une mutualisation des risques, tels l'accès à un travail décent et à la sécurité du revenu ${ }^{37}$. Le droit du travail répartit ces risques entre les travailleurs, les employeurs et les autres acteurs.

La législation sur les migrations insère les « travailleurs migrants » dans la répartition des risques comme un autre groupe d'intérêts, à côté des travailleurs locaux. La partie ouvrière de l'équation est donc divisée en deux, ayant dans certains cas des intérêts contradictoires. Les travailleurs locaux d'une part - nationaux comme migrants permanents - et les migrants d'autre part, deviennent des concurrents sur le marché du travail. Ce fait met en exergue un conflit d'intérêts au cœur du droit du travail : quel travailleur protéger? Le principe d'égalité et de non-discrimination reste l'un des principes fondamentaux et réglemente parallèlement le statut juridique des travailleurs migrants. Ce principe sert non seulement à protéger les travailleurs migrants sur le marché du travail, mais aussi à empêcher la concurrence déloyale vis-à-vis des travailleurs locaux, protégeant ainsi la main-d'œuvre locale.

32 J. Fudge, «Migrant Domestic Workers in British Columbia, Canada Unfreedom, Trafficking and Domestic Servitude », in J. Howe et R. Owens, Temporary Labour Migration in the Global Era, op. cit., p. 151.

33 H. Shamir, «A Labor Paradigm for Human Trafficking », UCLA Law Review, 60, 2012, p. 76.

34 lbid.

35 M. Freedland et C. Costello, « Migrants at Work and the Division of Labour Law », Migrants at Work, op. cit., p. 1.

36 M. Freedlandet et N. Kountouris, The Legal Construction of Personal Work Relations, Oxford Monographs on Labour Law, 2011, p. 439.

37 Ibid. 
Freedland et Kountouris suggèrent trois types de risques dans la régulation des relations personnelles de travail: la mutualisation verticale des risques (répartition des risques entre les employeurs et les travailleurs), la mutualisation horizontale des risques (répartition et redéploiement des risques parmi les travailleurs), et enfin la mutualisation en diagonale (allocation de risques réorganisée par l'introduction d'intermédiaires ${ }^{38}$ ). Les exemples des deux premiers cas seront examinés ci-après.

Selon l'une des pratiques établies, les migrants doivent déposer leurs demandes de permis de travail, du moins pour la première fois, depuis leur propre pays. Cependant, de nombreux dispositifs légaux, comme les quotas générés par les besoins économiques du marché du travail, visent à protéger les travailleurs locaux contre la concurrence des migrants ${ }^{39}$. Ainsi, s'agissant des migrants en vue d'occuper un emploi, les États-nations s'assurent, en exigeant une offre d'emploi ou un contrat de travail, que les migrants sont bien recrutés dans des secteurs où la pénurie de main-d'œuvre est avérée, protégeant de ce fait les travailleurs locaux. En conséquence, ces législations répartissent les risques entre les migrants d'une part et les travailleurs locaux d'autre part, et sont mises en œuvre principalement en faveur des travailleurs locaux.

Si un tel privilège semble justifiable, dans une certaine mesure, en ce qui concerne les immigrés en vue d'occuper un emploi, il en est différemment s'agissant des migrants forcés. Les migrants forcés n'ont en effet aucun droit légal d'accès au travail. Ceux sont donc les plus vulnérables qui supportent la plus grande partie du risque. Cela montre également que les situations particulières des migrants devraient être prises en compte pour parvenir à une mutualisation équitable du risque.

La législation sur les migrations vise à garantir que les migrants se présentant aux frontières occuperont effectivement l'emploi pour lequel ils sont autorisés à entrer. Pourtant, la législation sur les migrations peut conférer aux employeurs des pouvoirs extracontractuels impactant la vie personnelle des migrants. L'éventail des régimes liés à l'employeur fonctionne à grande échelle. D'une part, il existe le système de la « kafala » dans les pays membres du Conseil de Coopération du Golfe, qui prévoit qu'un promoteur est tenu de prendre la pleine responsabilité économique et juridique du travailleur migrant. À l'autre bout du spectre, il existe des régimes de visas liés à une profession, à un secteur ou à un employeur. Les travailleurs temporaires et saisonniers travaillent généralement sous un régime de visas liés. Dans les cas où le permis de travail du migrant est attaché à un seul employeur, le travailleur migrant n'aura pas le droit de mettre un terme à une relation contractuelle sans prendre le risque d'être expulsé du pays. Ces règles ont pour effet d'habiliter l'employeur, au-delà de la structure contractuelle, et de faire peser des risques supplémentaires sur les travailleurs migrants. 


\section{III - REPENSER LES RÉPONSES DU DROIT DU TRAVAIL}

L'une des principales raisons de la vulnérabilité des migrants en situation irrégulière sur le marché du travail est l'absence de droit au travail. Du point de vue du droit du travail, on peut douter qu'il y ait une justification raisonnable pour expliquer l'écart existant entre le droit au travail et le droit de séjour. Le caractère vague du droit au travail conduit à camoufler le fait que la citoyenneté prévaut comme condition préalable au droit au travail.

L'article 23 de la Déclaration universelle des droits de l'homme stipule que toute personne a droit au travail, comme au libre choix de son travail. Le Pacte international relatif aux droits économiques, sociaux et culturels proclame que «Les États parties au présent Pacte reconnaissent le droit au travail, qui comprend le droit de toute personne à obtenir la possibilité de gagner sa vie par un travail librement choisi ou accepté, et prendront des mesures appropriées pour sauvegarder ce droit » (article 6/1).

Le Comité des droits économiques, sociaux et culturels, qui donne une analyse systématique du droit au travail, énonce la nature générale de ce droit sous le titre «Travailleurs migrants et droit au travail ». Dans son observation générale n 18, le Comité donne un aperçu très complet du droit au travail combinant ces différents éléments ${ }^{40}$. Toujours selon le Comité, les États sont tenus de respecter le droit au travail en s'abstenant de nier ou de limiter l'égalité d'accès au travail décent pour toutes les personnes, particulièrement les personnes et groupes défavorisés ou marginalisés, y compris les travailleurs migrants ${ }^{41}$. Les travailleurs migrants devraient dès lors avoir un accès égal à un travail décent.

Le travail est le principal moyen de générer un revenu, condition cruciale pour répondre aux besoins les plus fondamentaux ${ }^{42}$. Si le droit au travail offre les conditions préalables indispensables à d'autres nécessités de la vie, c'est qu'il existe un lien étroit entre les libertés civiles et politiques, et le droit au travail ${ }^{43}$. En outre, le travail est un facteur d'estime de soi, d'estime pour les autres, d'auto-développement ou de réalisation de soi ${ }^{44}$. Le Comité des droits économiques, sociaux et culturels déclare que le droit au travail est essentiel pour la réalisation d'autres droits de l'homme et constitue une partie intégrante de la dignité humaine. Chaque individu a le droit de pouvoir travailler lui permettant ainsi de vivre dignement ${ }^{45}$.

40 Committee on Economic, Social and Cultural Rights, General Comment $n^{\circ} 18$, The Right to Work (24 novembre 2005, E/C12/GC/18); about the General Comment, voir C. O'Cinneide, "The Right to Work in International Human Rights Law», The Right to Work, Legal and Philosophical Perspectives, 2015, p. 99.

41 Committee on Economic, Social and Cultural Rights, General Comment $n^{\circ} 23$.

42 V. Mantouvalou, "Introduction», The Right to Work, Legal and Philosophical Perspectives, Hart Publishing, 2015, p. 1.

43 H. Collins, «Is There a Human Right to Work », in V. Mantouvalou (dir.), The Right to Work, Legal and Philosophical Perspectives, Hart Publishing, 2015, p. 18.

44 Voir G. Mundlak, "The Right to Work - The Value of the Work», in D. Barak-Erez et A. M. Gross (dir.), Exploring Social Rights, Between Theory and Practice, Bloomsbury Collections, 2011, p. 341; H. Collins, «Is There a Human Right to Work», op. cit., p. 17.

45 Committee on Economic, Social and Cultural Rights, General Comment n 18, para. 1. 
La Cour européenne des droits de l'homme a également montré des liens étroits entre le droit au travail et le droit au respect de la vie privée et familiale, considérant de ce fait que l'exclusion du marché du travail est une sanction qui exige une justification au regard de l'article 8 de la CEDH ${ }^{46}$.

Si la capacité financière et technique des États est limitée pour créer des emplois en faveur de ceux qui en souhaitent un, l'objectif est en revanche plus facile à atteindre en ce qui concerne l'aspect négatif du droit au travail: le droit pour chacun de gagner sa vie par un travail décent. Les migrants sont non seulement privés de cet aspect fondamental du droit au travail mais aussi, dans certains cas, exclus du droit d'avoir un travail décent dans le pays d'accueil (qu'il s'agisse des migrants entrés de manière irrégulière ou de ceux qui ont un droit de séjour légal). Rien, pas même la protection des travailleurs locaux, ne semble pouvoir justifier l'exclusion des migrants du marché du travail et de son droit afférent: la liberté d'occuper durablement un emploi. Ceux qui ont le droit de séjourner dans un pays étranger ont aussi des droits fondamentaux, y compris le développement et l'accomplissement de la personnalité qui font partie intégrante du droit à la vie privée.

Les instruments du droit du travail relatifs à la validité du contrat de travail et aux différents types du contrat de travail peuvent également contribuer à remédier à certaines vulnérabilités des travailleurs migrants.

Dans certains cas, les institutions juridiques censées être plus protectrices en matière de droits du travail peuvent avoir des effets néfastes en ce qui concerne les migrants. A titre d'exemple, Herzfeld Olsson soutient qu'un type particulier de contrat de travail - en l'occurrence le contrat à durée déterminée - auquel il ne peut être mis fin que pour des raisons légitimes ou en cas de négligence grave des migrants, va fournir aux migrants la sécurité d'emploi pendant la durée de leur permis de travail47.

De même, la syndicalisation des travailleurs migrants, parfois extrêmement mobiles, exige des changements dans la structure et les stratégies des syndicats ${ }^{48}$. Les syndicats deviennent donc des acteurs importants dans la prévention de la traite des êtres humains et du travail forcé ${ }^{49}$. Les droits collectifs du travail constituent un outil juridique important pour améliorer les conditions de travail.

46 H. Collins, "Progress Towards the Right to Work in UK», in V. Mantouvalou (dir.), The Right to Work, Legal and Philosophical Perspectives, op. cit., p. 250.

47 P. Herzfeld Olsson, "Empowering Temporary Migrant Workers in Sweden A Call for Unequal Treatment », in J. Howe et R. Owens (dir.), Temporary Migration in the Global Era, 2016, p. 203.

48 I. Greer, Z. Ciupijus, "The European Migrant Workers Union and the Barriers to Transnational Industrial Citizenship », European Journal of Industrial Relations, 19 (1), 2013, p. 5.

49 Concernant le Projet d'action tripartite de I'OIT visant à protéger les travailleurs migrants de la sous-région du Grand Mékong contre l'exploitation du travail, voir E. Marks \& A. Olsen, "The Role of Trade Unions in Reducing Migrant Worker Vulnerability to Forced Labour and Human Trafficking in the Greater Mekong Subregion », Anti-Trafficking Review, n 5, 2015, p. 111, www.antitraffickingreview.org. 


\section{PROTÉGER LES DROITS DES TRAVAILLEURS MIGRANTS}

\section{Conclusion}

"Le travail des migrants n'est pas une marchandise ", comme l'indiquent Kountouris et Freedland ${ }^{50}$. La migration comme phénomène mondial de justice sociale met en exergue les difficultés relatives aux frontières et aux institutions du droit du travail. Les États-nations préfèrent réglementer la migration au service des besoins spécifiques de leurs marchés du travail respectifs, et tentent d'éviter les migrants forcés. Le statut juridique des travailleurs migrants se forge à l'intersection du droit des migrations, du droit du travail, du droit international et du droit pénal. Incontestablement, les travailleurs migrants posent aujourd'hui de nouveaux défis au droit du travail. Pour mettre en œuvre le principe de l'égalité entre les migrants et les ressortissants au regard des droits du travail et de la sécurité sociale, le droit du travail devrait développer une approche spécifique, qui permettrait d'éviter les vulnérabilités et l'exploitation sur le marché, et de renforcer les institutions du droit du travail.

50 N. Kountouris, M. Freedland, «Epilogue Resocialising Europe - Looking Back and Thinking Forward », Resocialising Europe in a Time of Crisis, 2013, p. 493.

\section{KÜBRA DOGAN YENISEY}

Professeur de droit du travail et de la sécurité sociale, Université d'Istanbul Bilgi, Faculté de droit.

Thématiques de recherche: Travailleurs migrants, modèles de négociation collective, gig-économie et droit du travail, harmonisation du droit turc avec celui de l'UE.

\section{Publications:}

K.Dogan Yenisey, (with B. Ceylan Ataman), « Decentralised Collective Bargaining: A Solution to Economic Crisis? - The Case of Turkey - ", Collective Bargaining Developments in Times of Crisis, ed. by S. Laulom, Bulletin of Comparative Labor Relations, 2018.

$\sim$ K. Dogan Yenisey, "Safeguarding the Rights of Migrant Workers: Another Challenge for Labour Law? », in G. Casale et T. Treu (dir.), Transformations of Work - Challenges for Institutions and Social Actors, Bulletin of Comparative Labour Relations, Kluwer Law International, 2019. 